\title{
Visual recognition as a function of stimulus offset asynchrony and duration*
}

\author{
LEE S. COHENE $\dagger$ and HAROLD P. BECHTOLDT \\ University of Iowa, Iowa City, Iowa 52242
}

\begin{abstract}
The stimuli consisted of two complementary dot patterns that formed a bigram when they were flashed simultaneously; impairment of letter recognition developed when one of the patterns was briefly extended beyond the termination of the other (stimulus of fset asynchrony). However, if the ratio of stimulus offset asynchrony to bigram duration remained constant, the probability of a correct recognition response also remained constant as duration varied over a 50- to $100-\mathrm{msec}$ interval. When percent stimulus asynchrony increased, the impairment increased. An interaction between bigram letter position and each of bigram duration and percent stimulus asynchrony was observed with recognition accuracy greater in general for the letter in the left half of the field.
\end{abstract}

Two independent variables which appear quite regularly in masking studies are, first, the temporal onset or temporal offset differences known as the stimulus asynchrony effect and, second, the duration of the stimulus presentation in the recognition task (Kahneman, 1968; Turvey, 1973). Of the two temporal asynchrony manipulations, it appears that the perhaps more important and less well investigated is the one that deals with the time of offset rather than the time of onset of two stimuli having certain temporal durations. The present study is thus concerned with the two independent variables of stimulus offset asynchrony and stimulus duration.

Of the many theories which have been formulated about masking phenomena, two which have received considerable attention are Sperling's $(1960,1963,1971)$ erasure hypothesis and Eriksen and Collins's (1967, 1968 ) integration hypothesis. In general, Sperling (1963, 1965,1971 ) assumes that a masking stimulus effectively halts all processing of the target stimulus that is presumably taking place in a theoretical short-term visual storage system. Therefore, whatever has not yet been transferred to short-term (or long-term) memory is lost. As Haber (1969) points out, no mechanism was proposed for how this interference occurred.

Strong support for an integrative process associated with visual masking phenomena has been provided by Eriksen and Collins $(1967,1968)$. This integration has been investigated with pairs of temporally separated dot pattern stimuli such that the neural trace arising from the first pattern must be combined with the neural activity of the second pattern for a verbal recognition

\footnotetext{
*This paper is based on a thesis completed by the first author under the direction of the second author and submitted to the University of Iowa in partial fulfillment of the requirements of the MA degree. We wish to thank the anonymous reviewers for their helpful suggestions.

$\nmid$ Requests for reprints should be sent to Lee S. Cohene, Department of Psychology, University of Iowa, Iowa City, Iowa 52242.
}

response to occur. The two dot patterns, when combined by superimposition, form an easily recognized nonsense syllable. According to Eriksen (e.g., Eriksen \& Rohrbaugh, 1970), masking may occur between two stimuli because the combination of a second stimulus with the first usually forms a pattern too complex for recognition. However, when the stimuli complement each other (Eriksen \& Collins, 1967, 1968), integration permits recognition of the syllable,

Eriksen and Collins (1967, Experiment 2) summarized their results as indicating that the ability to organize the nonsense syllables from two separate stimulus halves was: (a) greatest when the stimulus halves were concurrent and decreased as the halves were separated in time; (b) highest when the stimulus halves were of equal energy (duration) and decreased in a negatively accelerated function as the mismatch in energy increased; and (c) indifferent to the order of occurrence of unequal energy stimulus halves, except when the occurrence of the stimulus halves overlapped in time (concurrent). In the latter instance, performance was best if the unequal duration stimulus halves shared a common offset rather than a common onset, and the greater the inequality in durations, the greater was the interference effect. The authors interpreted their data as supporting the assumption that inequality in energy between stimulus halves reduces integration of the embedded nonsense syllable.

The present experiment is concerned with the only case in the Eriksen and Collins (1967) study where order of occurrence of long and short duration halves made a difference; this situation was the one in which the halves were concurrently on for the duration of the short half. And in this instance, the result appears to be a consequence of the fact that the long-half second group showed a conspicuously rapid decline in recognition that was quite deviant from the pattern of results obtained in the other conditions.

As Eriksen and Collins (1967) commented, "This finding is in itself puzzling [p. 483]." It does not fit a 
(a)

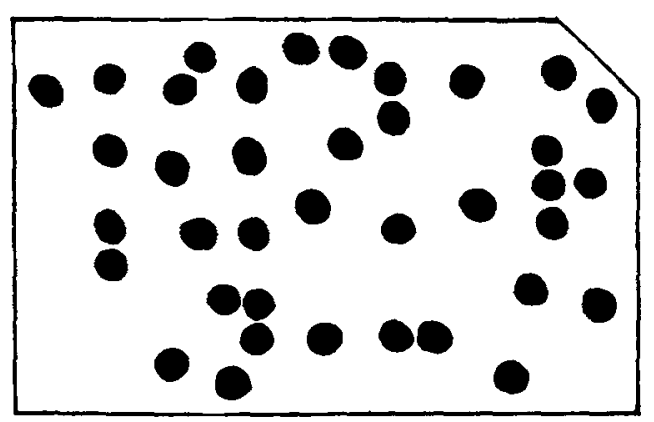

(b)

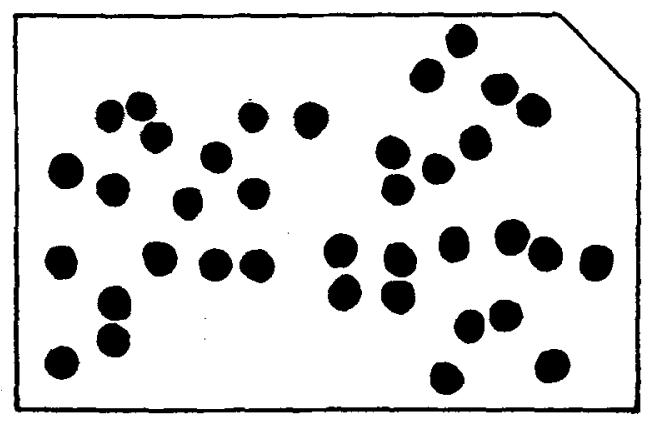

(c)

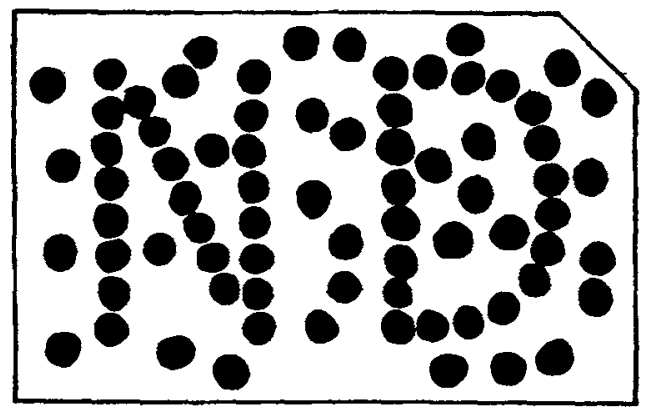

Fig. 1. Two complementary dot pattern stimuli (a, b) which form a recognizable bigram composite (c) when one dot pattern is superimposed on the other.

trace decay theory, since both halves always shared a common 25-msec duration. Performance under either order was poorer than if both halves had been presented for only $25 \mathrm{msec}$ concurrently. The inequality of the energy in the stimulus halves could possibly account for the decreased performance, except that the inequality should have been the same for either order of the halves' occurrence.

The study reported below was consequently designed to determine, first, whether the important divergent findings of Eriksen and Collins (1967) were reproducible with new stimuli and new procedures. In addition, the concurrent, long-half second (simultaneous onset) paradigm using dot pattern stimuli is especially appropriate for an investigation of the integration-interruption distinction. Since integration of the stimulus halves has been demonstrated by. Eriksen and Collins (1967) with this type of stimulus at one concurrent interval of $25 \mathrm{msec}$, it is important to determine both whether such integration can also be found at longer stimulus durations and whether the "puzzling" (interference) result of the initial study (where "integration" was perhaps "interrupted") is also obtained at other asynchrony intervals as the stimulus overlapping interval is increased. Such findings would indicate that both integration and interference processes may be required to explain performance in stimulus paradigms involving different temporal onset and offset asynchrony as well as different stimulus duration.

\section{METHOD}

\section{Subjects}

The Ss were 110 students ( 44 females and 66 males) from the introductory psychology course at the University of lowa. Ninety of these students were randomly assigned upon arrival at the experiment to one of nine groups ( $10 \mathrm{Ss}$ per group). Midway through the main experiment, two groups of $10 \mathrm{Ss}$ each were added to the experiment, again with random assignment.

\section{Materials and Apparatus}

The "composite" stimuli consisted of 10 letters which formed the following 15 bigrams: $\mathrm{BH}, \mathrm{BK}, \mathrm{DH}, \mathrm{DV}, \mathrm{FB}, \mathrm{HO}, \mathrm{KO}, \mathrm{KS}$, ND, NR, OV, RF, SF, SN, and VR.

The letters were composed of nonoverlapping black dots on a white background, as shown by the sample stimulus in Fig. 1c. The dots making up the forms of the letters were divided over two complementary stimulus halves, $S 1$ and $S 2$. As demonstrated by Cohere and Bechtoldt (1972b), little information as to the nature of the nonsense syllable composite was available from either half alone (Figs. $1 \mathrm{a}$ and $1 \mathrm{~b}$ ). To reduce cues further, camouflaging dots of equal size and distribution were scattered over each complementary half. The stimulus halves, photographed on $35-\mathrm{mm}$ high-contsast positive transparencies, were mounted in stereo Realist slides for projection by a Compco Stereo 500 projector. Control of the on-off exposure durations and sequences was by solid-state circuitry.

The dot patterns were projected onto a rear projection Polacoat screen. The projected pattern frame was $23.3 \mathrm{x}$ $15.0 \mathrm{~cm}$, with the projected bigram covering $17.6 \times 11.5 \mathrm{~cm}$ and 3.9 to $4.7 \mathrm{~cm}$ separating the two letters. The dots were approximately $1.2 \mathrm{~cm}$ in diam. The framed pattern subtended 26.2 deg of visual angle in width and $17.3 \mathrm{deg}$ in height.

At the point of S's eyes, the room light transmitted through the screen was $0.5 \mathrm{fc}$. The light transmitted by one projector alone was $0.6 \mathrm{fc}$ and by both projectors together was $0.7 \mathrm{fc}$.

The S's chin rested in an adjustable chinrest, and a shield, which was manually operated from E's location, was positioned so as to block S's view of the screen. A large card listing the set of 10 letters was placed in front of $S$ at the base of the screen so as to be visible when the shield was either up or down.

\section{Procedure}

The original design was a 3 by 3 by 2 factorial combination of two between-S variables and a trials variable. One between-S variable (bigram duration) was the duration $(50,75$, and $100 \mathrm{msec}$ ) used for the simultaneous exposure of the two complementary dot patterns, S1 and S2. The other between-S variable (stimulus asynchrony) was the difference in duration between $S 1$ and S2 expressed as a percent $(0 \%, 20 \%$, and $40 \%$ ) of the exposure duration value for the composite pattern. The $0 \%$ condition served as a control (simultaneous onset and offset) for the $20 \%$ and $40 \%$ conditions; for the $20 \%$ and $40 \%$ 
conditions, the S2 pattern presentations continued after the S1 pattern was terminated. For the trials variable, $S s^{\prime}$ responses for four trials of 15 presentations each were recorded in blocks of two trials yielding two blocks per $\mathrm{S}$.

Two control groups ( 35 and $150 \mathrm{msec}$ duration) were added to the bigram duration variable with $0 \%$ asynchrony midway through the experiment because little improvement had been observed in the 50 to $100-\mathrm{msec}$ range for this condition. The lower value of $35 \mathrm{msec}$ was selected because it was close to the recognition threshold previously obtained with similar stimuli (Cohene \& Bechtoldt, 1972a, b). The 150-msec value was chosen as a reasonable upper value.

The order of slide presentations was randomly determined with each slide being presented once during each trial. A forced-choice recognition procedure based upon the set of 10 letters was employed, and $\mathrm{Ss}^{\prime}$ correct and incorrect bigram responses were recorded. The Ss were not told how many bigrams were in the set. However, they were informed that no double letters would appear in any presentation.

A ready signal was delivered verbally 2 to $4 \mathrm{sec}$ prior to each presentation. At the same time that the signal was given, the shield blocking S's view was lifted. Each S was instructed to fixate on a point, during the 2- to $4-\mathrm{sec}$ interval, which was approximately midway between the letters of the bigram that followed. The shield was replaced about $1 \mathrm{sec}$ following S's response. The interval between presentations was approximately 10 to $20 \mathrm{sec}$ (with an average of $15 \mathrm{sec}$ ), during which time S's response was recorded and the subsequent slide was focused.

Six trials of the 15 different bigram stimuli were actually administered to each $S$, with the first two trials providing a practice sequence. During these first two trials only, Ss were informed as to the number of letters they had correctly identified (i.e., either both letters, one letter, or none) following each presentation.

\section{RESULTS}

The mean number of correct letter recognition responses for the trial blocks are presented in Table 1. Analysis of the mean differences in the original design of Bigram Duration $(50,75$, and $100 \mathrm{msec})$ by Stimulus Asynchrony $(0 \%, 20 \%$, and $40 \%)$ by Trial Blocks (B1 and B2) yielded no duration effect, but the main effects for both stimulus asynchrony $[\mathrm{F}(2,81)=124.78$, $\mathrm{p}<.05]$ and for blocks $[\mathrm{F}(1,81)=37.29, \mathrm{p}<.05]$ were significant. The interactions were not significant. A

\section{Table 1}

Mean Number of Correct Letter Recognition Responses for Blocks (B1 and B2) of Two Trials Each as a Function of Bigram Duration and Percent Stimulus Asynchrony

\begin{tabular}{|c|c|c|c|c|c|c|}
\hline \multirow{3}{*}{$\begin{array}{l}\text { Bigram } \\
\text { Duration } \\
\text { (msec) }\end{array}$} & \multicolumn{6}{|c|}{ Stimulus Asynchrony } \\
\hline & \multicolumn{2}{|c|}{$0 \%$} & \multicolumn{2}{|c|}{$20 \%$} & \multicolumn{2}{|c|}{$40 \%$} \\
\hline & B1 & B2 & B1 & B2 & B1 & B2 \\
\hline 35 & 33.3 & 33.4 & -- & -- & -- & -- \\
\hline 50 & 36.8 & 40.6 & 29.4 & 32.2 & 17.3 & 20.1 \\
\hline 75 & 42.1 & 45.1 & 32.4 & 34.0 & 18.6 & 18.8 \\
\hline 100 & 43.3 & 46.3 & 31.3 & 33.0 & 15.1 & 19.4 \\
\hline 150 & 49.0 & 51.6 & - & -- & $\ldots$ & -- \\
\hline
\end{tabular}

Note-The error mean square between [MS(b)] and the error mean square within [MS(w)] are 76.077 and 6.877, respectively, for the five bigram duration conditions with $0 \%$ stimulus asynchrony. The $M S(b)$ and $M S(w)$ are 70.609 and 8.019, respectively, for the 50-, 75., and 100-msec bigram duration conditions with all conditions of stimulus asynchrony.
Table 2

Mean Number of Correct Letter Recognition Responses for Left (L) and Right (R) Letter Positions as a Function of Bigram Duration and Percent Stimulus Asynchrony

\begin{tabular}{|c|c|c|c|c|c|c|}
\hline \multirow{3}{*}{$\begin{array}{c}\text { Bigram } \\
\text { Duration } \\
\text { (msec) }\end{array}$} & \multicolumn{6}{|c|}{ Stimulus Asynchrony } \\
\hline & \multicolumn{2}{|c|}{$0 \%$} & \multicolumn{2}{|c|}{$20 \%$} & \multicolumn{2}{|c|}{$40 \%$} \\
\hline & L & $\mathbf{R}$ & $\mathbf{L}$ & $\mathbf{R}$ & $\mathbf{L}$ & $\mathbf{R}$ \\
\hline 35 & 32.9 & 33.8 & -- & -- & -- & -- \\
\hline 50 & 41.2 & 36.2 & 29.9 & 31.7 & 18.2 & 19.2 \\
\hline 75 & 49.0 & 38.2 & 34.9 & 31.5 & 20.3 & 17.1 \\
\hline 100 & 49.0 & 40.6 & 34.6 & 29.7 & 20.4 & 14.1 \\
\hline 150 & 52.8 & 47.6 & -- & -- & -- & -- \\
\hline
\end{tabular}

Note-The error mean square between [MS(b)] and the error mean square within $[M S(w)]$ are 76.077 and 29.894, respectively, for the five bigram duration conditions with $0 \%$ stimulus asynchrony. The $M S(b)$ and $M S(w)$ are 70.609 and 38.547 , respectively, for the 50, 75-, and 100-msec bigram duration conditions with all conditions of stimulus asynchrony.

follow-up analysis of the differences between the stimulus asynchrony means (averaged across duration and blocks yielding a maximum possible score of 60 ) indicated that all pairwise differences among the means $(0 \%=42.4,20 \%=32.0$, and $40 \%=18.2)$ were reliable in terms of a Scheffé criterion.

An analysis of the $0 \%$ asynchrony data with all five bigram durations $(35,50,75,100$, and $150 \mathrm{msec})$ on the trial blocks (B1 and B2) indicated a statistically significant duration effect $[F(4,45)=10.84, p<.05]$ as well as a practice effect $[F(1,45)=22.71, p<.05]$. Again, the interactions were not significant. As expected, the significant duration effect arose as a result of adding the 35 - and $150-\mathrm{msec}$ conditions to the design. The mean number of correct recognition responses averaged across blocks ( 60 as the possible score) for the five durations were $35 \mathrm{msec}=33.4,50 \mathrm{msec}=38.7$, $75 \mathrm{msec}=43.6,100 \mathrm{msec}=44.8$, and $150 \mathrm{msec}=50.3$. In a test of the pairwise comparisons of these means, only the difference between 35 and $150 \mathrm{msec}$ was reliable (Scheffé criterion).

Similar analyses were carried out with bigram letter position (left and right), instead of blocks, as the within-S variable. For the analyses of the effect of letter position, the data were summed over the two trial blocks. The mean number of correct letter recognition responses for letter position are listed in Table 2.

An analysis of variance of Bigram Duration by Stimulus Asynchrony by Letter Position resulted in a statistically significant position main effect $[F(1,81)=$ 22.15, $\mathrm{p}<.05]$ as well as significant Position by Duration $[\mathrm{F}(2,81)=3.88, \mathrm{p}<.05]$ and Position by Asynchrony $[F(2,81)=4.06, p<.05]$ interactions. The triple interaction was not significant. (The insignificant duration effect and significant asynchrony effect were consistent with the previously reported blocks effect analysis.) A follow-up analysis of the simple position effects using the stimulus asynchrony means (averaged across duration and blocks) was carried 
out at both the left position (with means of $0 \%=46.4$, $20 \%=33.1$, and $40 \%=19.6)$ and the right position (with means $0 \%=38.3,20 \%=31.0$, and $40 \%=16.8$ ). All the pairwise differences of these simple effects were reliable.

The analysis of the $0 \%$ asynchrony data for letter position with all five bigram durations indicated a significant duration effect as in the blocks effect analysis, a significant position effect $[F(1,45)=27.17$, $\mathrm{p}<.05$ ] and a significant Position by Duration interaction $[F(4,45)=3.25, p<.05]$. As implied by this interaction, the trends for the left and right positions as a function of duration are not parallel. The left letter position mean numbers of recognitions were $35 \mathrm{msec}=$ $32.9,50 \mathrm{msec}=41.2,75 \mathrm{msec}=49.0,100 \mathrm{msec}=49.0$, and $150 \mathrm{msec}=52.8$. An analysis of these means using a Scheffé criterion showed that the differences between the value for $35 \mathrm{msec}$ and those for 75,100 , and $150 \mathrm{msec}$ were reliable. In addition, the difference (11.6) between 50 and $150 \mathrm{msec}$ closely approached the critical value. For the right letter position, the mean number correct for $35 \mathrm{msec}=33.9,50 \mathrm{msec}=36.2$, $75 \mathrm{msec}=38.2,100 \mathrm{msec}=40.6$, and $150 \mathrm{msec}=47.6$. Only the difference between 35 and $150 \mathrm{msec}$ exceeded the Scheffé critical value.

The results of the analyses of the test block data may be summarized in the following manner. In the original Duration by Asynchrony by Trials design using nine groups, for each of three levels of percent stimulus asynchrony, the probability of a correct recognition response is constant, regardless of bigram duration. For the original range of stimulus duration tested $(50-100 \mathrm{msec})$, if the duration of $S 2, D(S 2)$, is equal to or greater than the duration of $S 1, D(S 1)$, then the probability of a correct recognition response is constant for each ratio relating stimulus asynchrony to bigram duration, i.e., $[D(S 2)-D(S 1)] / D(S 1)$, at each level of duration. Thus, no differences were observed among the $0 \%$ asynchrony groups, the $20 \%$ asynchrony groups, or the $40 \%$ asynchrony groups; however, the groups with less asynchrony indicated better recognition than did the groups with greater asynchrony. Only when the 35 - and 150-msec conditions were added did recognition appear to improve with increasing duration. Recognition performance also increased with practice at a nearly constant rate over the three durations.

The results indicated a letter position difference favoring the left position of the bigram in general. Although a small difference favoring the right position appeared at the shortest duration $(35 \mathrm{msec})$ with $0 \%$ asynchrony, an increasing difference in recognition favoring the left position was obtained with increases in duration between 35 and $75 \mathrm{msec}$; thereafter, the difference decreased slowly with further increases in duration. A somewhat similar pattern of results was observed in the data for the nine groups of the Duration by Asynchrony by Position analysis, with an increasing difference between 50 and $75 \mathrm{msec}$ and then leveling off so that no change was observed at $100 \mathrm{msec}$. It was also demonstrated that recognition of the left position letters was superior to recognition of the right letters at $0 \%$ asynchrony for durations of $50 \mathrm{msec}$ or more. The rate of increasing impairment for left letters was constant and rapid as percent asynchrony increased to $20 \%$ and $40 \%$. The right position letters showed a much slower, though significant, rate of decline in recognition from $0 \%$ to $20 \%$ asynchrony, but thereafter showed a rate of increasing impairment similar to that for left position letters.

\section{DISCUSSION}

In line with the results of Eriksen and Collins (1967), an enormous impairment in recognition performance was obtained as the stimulus asynchrony increased. This impairment was observed when the concurrent exposure of the two stimulus components, S1 and S2, ranged from 50 to $100 \mathrm{msec}$, whereas Eriksen and Collins (1967) used a duration of only 25 msec. Furthermore, a direct and simple functional relationship relating the impairment to stimulus asynchrony (difference in duration between $\mathrm{S} 1$ and $\mathrm{S} 2$ ) and bigram duration (simultaneous exposure duration of S1 and S2) was suggested. While the parameters of the function found in the present experiment are not consistent with the results of Eriksen and Collins (1967), the same type of decreasing performance function was observed.

The results also suggest the possible introduction of set and attention variables which should be systematically varied in future experiments. The left letter position superiority of the bigrams was totally consistent with the usual letter recognition results of tachistoscopic presentation experiments in which this outcome is usually thought to be a function of reading habits, i.e., an attention variable (Moray, 1970). It may be that a set variable was also involved. That is, although Ss were instructed to fixate in the center of the screen (between the two letters), they may have oriented themselves towards the left visual field as a result of the instructions to report the left letter first. Finally, it was observed that this left position superiority interacted with both bigram duration and percent stimulus asynchrony. The importance of set and attention factors in the masking paradigm has been emphasized by Eriksen and his associates (Eriksen \& Collins, 1969; Eriksen \& Hoffman, 1972; Eriksen \& Lappin, 1967).

A question may be posed concerning the interpretation of these data in terms of masking concepts. Consider the operations that gave rise to the results. Two dot patterns, S1 and S2, were simultaneously flashed with a concurrent onset to form the target stimulus (TS), but S2 remained on for a brief duration following S1 offset to create the "masking" stimulus (MS). This is seen by Kahneman (1968) as one of the general limiting cases of visual masking. Here, masking is paradigmatically defined. The mechanism, 
however, is as yet unaccounted for and conceivably may be quite different from any that has been proposed to date for "masking" results. Kahneman (1968), for one, has argued that the mechanisms underlying the limiting cases of visual masking may be quite different from other cases and from each other.

Another feature of the current experiment relevant to the analysis of the factors operating was that the impairment in recognition performance may be viewed as a result of "subtraction" from the TS rather than as an addition of MS. During each presentation, the termination (subtraction) of S1 from the total stimulus configuration necessarily meant the termination of the bigram presentation, but because no new or added stimulus followed, it is difficult to predict impairment of recognition on the basis of either "erasure" or "integration." The stimulus following S1 offset was simply the continuation of S2. According to the statements of the "erasure" theory, the bigram should already have been transferred to a short-term memory or encoded in some way within the period of 50 to $100 \mathrm{msec}$, even if the continuation of S2 were considered to be a "new" stimulus; then the bigram should avoid "erasure" (Sperling, 1963, 1971). It appears that either the parameters of the erasure theory or the applicability of the analysis in terms of the encoding of an icon must be altered to account for the present results.

An attempt to account for the impaired recognition using an integration formulation, as given by Eriksen and his associates, might follow the line of luminance summation. However, because the luminance of $S 1$ and S2 was very near to that of the adapting field (which was considered to be the brightness of the screen during the absence of stimulus presentation) and because the luminance of $S 1$ equalled that of S2, the amount of luminance summation would seem to be negligible. Further, the contribution of any type of energy variable to the recognition performance variance in the present study also seems minimal, since there was so little variation in mean accuracy of recognition in the $0 \%$ asynchrony groups in the range of $50-$ to $100-\mathrm{msec}$ exposure. For these reasons, an appeal to unequal energy ratios, in terms of a time-intensity reciprocity theory, as the explanation for the striking interference effects of the present study is hardly justified. Since it is conceivable that unequal energy ratios over a wider range of values could produce similar masking effects, further work directed to the influence of the factor of energy ratios would be valuable.

Another possible type of "integration" is the combination of the trace of the bigram with that of the following S2 dot pattern, the combination resulting in contour interaction. This explanation does not seem to be an acceptable one for several reasons. In the first place there is no physical contour in the usual sense; the dots forming the pattern are clearly separated from each other. Further, the S2 extension had already constituted a part of the bigram (during S1-S2 simultaneous presentation); one does not expect to find contour interaction between an intelligible stimulus pattern and a part of itself, although this possibility has not received any experimental consideration to date.

Although Sperling's erasure hypothesis has been dismissed as an explanation of the data, a more general form of the interruption formulation as suggested by several writers (Eriksen \& Collins, 1968; Lindsley, 1961; Turvey, 1973) may suffice. Simply stated, the hypothesis proposes that the extended S2 duration interrupts the transfer of a visually coded stimulus into a covert response [e.g., preventing the excitation of a cell assembly in Hebb's (1949) terms]. An alternative statement would be that the trace of the already-formed covert response is interrupted by the incoming S2 excitation (e.g., inhibition of the cell assembly) and then lost. Both of these postulates implicate interference of the response mechanism rather than an interaction of the neural traces of the stimuli on the sensory side of the process. One implication of this response-interruption hypothesis could be tested in a study similar to the one reported here, but with simultaneous offset ("forward masking") rather than simultaneous onset ("backward masking"); such a study would be an extension of another phase of the original Eriksen and Collins (1967) investigation. Eriksen and Collins (1967) found that, while recognition in the simultaneous-offset/variableonset conditions was much superior to recognition in the simultaneous-onset/variable-offset condition, recognition impairment which appeared to be positively correlated with temporal variation still occurred.

A comment should be made about the obtained trial effect. While Eriksen and Collins $(1967,1968)$ provided their Ss with an extended amount of prior experience with the stimuli so that an approximately $90 \%$ probability of response was achieved, the Ss in the present study received no such extensive practice. Their only exposure prior to testing consisted of two trials with minimal feedback. It is encouraging to note, then, the small amount of change in performance from Block 1 to Block 2 of the testing series as well as the generally adequate level of performance.

Another discrepancy between the study reported here and that of Eriksen and Collins (1967) involves the stimulus asynchrony parameters; whereas the present experiment indicated increasingly severe impairment of recognition in the range of $0 \%$ to $40 \%$ stimulus asynchrony, the range under observation in the Eriksen and Collins experiment was $0 \%$ to $600 \%$ asynchrony. Aside from differences in prior practice and instructions, this variation in performance may arise from the difference in stimuli. The stimuli in the present experiment were composed of black dots on a white ground and were enclosed in a visual field that required peripheral vision $(26.2 \times 17.3 \mathrm{deg})$. Eriksen and Collins, on the other hand, employed stimuli of white dots on a black ground that required only near-foveal vision ( $2 \mathrm{x}$ 
$1 \mathrm{deg}$ ). All of these task and stimulus differences point to the need for careful consideration of experimental operations and for an analysis tied closely to these operations.

\section{REFERENCES}

Cohene, L. S., \& Bechtoldt, H. P. Some temporal factors in visual pattern recognition. Paper presented at the annual meeting of the Iowa Academy of Science, Iowa City, Iowa, April 1972a.

Cohene, L. S., \& Bechtoldt, H. P. Some temporal factors in visual pattern recognition. II. Unpublished manuscript, University of lowa, $1972 b$.

Eriksen, C. W., \& Collins, J. F. Some temporal characteristics of visual pattem perception. Journal of Experimental Psychology, 1967, 74, 476-484.

Eriksen, C. W., \& Collins, J. F. Sensory traces versus the psychological moment in the temporal organization of form. Journal of Experimental Psychology, 1968, 77, 376-382.

Eriksen, C. W., \& Collins, J. F. Temporal course of selective attention. Journal of Experimental Psychology, 1969, 80, 254-261.

Eriksen, C. W., \& Hoffman, J. E. Some characteristics of selective attention in visual perception determined by vocal reaction time. Perception \& Psychophysics, 1972, 11, 169-171.

Eriksen, C. W., \& Lappin, J. S. Selective attention and very short-term recognition memory for nonsense forms. Journal of Experimental Psychology, 1967, 73, 358-364.

Eriksen, C. W., \& Rohrbaugh, J. Visual masking in multielement displays. Journal of Experimental Psychology, 1970, 83, 147-154.

Haber, R. N. (Ed.) Information-processing approaches to visual perception. New York: Holt, Rinehart \& Winston, 1969.

Hebb, D. O. Organization of behavior. New York: Wiley, 1949.

Kahneman, D. Method, findings, and theory in studies of visual masking. Psychological Bulletin, 1968, 70, 404-425.

Lindsley, D. B. Electrophysiology of the visual system and its relation to perceptual phenomena. In M. A. B. Brazier (Ed.), Brain and behavior. Vol. 1. Washington, D.C: American Institute of Biological Sciences, 1961.

Moray, N. Attention. New York: Academic Press, 1970.

Sperling, G. The information available in brief visual presentations. Psychological Monographs, 1960, 74(11, Whole No. 498).

Sperling, G. A model for visual memory tasks. Human Factors, $1963,5,19-31$.

Sperling, G. Temporal and spatial visual masking: I. Masking by impulse flashes. Journal of the Optical Society of America, $1965,55,541-559$.

Sperling, G. Information retrieval from two rapidly consecutive stimuli: A new analy sis. Perception \& Psychophysics, 1971, 9, 89-91.

Turvey, M. T. On peripheral and central processes in vision: Inferences from an information-processing analysis of masking with patterned stimuli. Psychological Review, 1973, 80, 1-52.

(Received for publication July 27, 1973;

revision received September 27, 1973; accepted September 28, 1973.) 